\title{
A model protocol evaluating the introduction of genetic assessment for women with a family history of breast cancer
}

\begin{abstract}
Jonathon Gray, Kate Brain, Paul Norman, Cathy Anglim, Liz France, Garry Barton, Lucy Branston, Evelyn Parsons, Angus Clarke, Julian Sampson, Elizabeth Roberts, Robert Newcombe, David Cohen, Cerilan Rogers, Robert Mansel, Peter Harper
\end{abstract}

Institute of Medical Genetics, University of Wales College of Medicine, Heath Park, Cardiff CF4 4XN, UK

J Gray

K Brain

C Anglim

E France

E Parsons

A Clarke

J Sampson

P Harper

Psychology

Department, Sheffield

University, Sheffield,

UK

P Norman

Health Economics,

University of

Glamorgan,

Glamorgan, UK

G Barton

D Cohen

Breast Test Wales, Cardiff, UK

L Branston

E Roberts

C Rogers

Department of Medical Computing and Statistics,

University of Wales

College of Medicine,

Cardiff, UK

R Newcombe

Department of

Surgery, University of

Wales College of

Medicine, Cardiff, UK

R Mansel

Correspondence to:

Dr Gray

Revised version received 28 April 1999

Accepted for publication

5 August 1999

\begin{abstract}
Randomised controlled trials allow comparisons to be made between different models of service delivery, but have not been used in the field of clinical genetics. With the advent of clinical governance, the evidence provided by such trials will be increasingly important in informing and shaping clinical genetics practice. The TRACE project (Trial of genetic assessment in breast cancer) is a randomised controlled trial of genetic assessment for women who are at increased risk of breast cancer because of their family history. The absence of cancer genetics service provision in Wales before this study gave a window of opportunity in which this important trial could be conducted. The present paper describes how TRACE will provide crucial evidence regarding the psychosocial as well as resource implications of adding individualised genetic assessment, genetic counselling, and (where appropriate) gene testing to typical advice and surveillance from a hospital breast clinic. In addition, it is anticipated that TRACE will represent a model for future trials of service delivery in the increasing number of complex genetic disorders where evidence on the economic implications of screening and management is currently limited.

(7 Med Genet 2000;37:192-196)
\end{abstract}

Keywords: genetic assessment; family history; breast cancer

Breast cancer is the commonest cancer in women in the UK. The incidence is among the highest in the world with approximately 30000 new cases presenting each year. Although most breast cancer arises in women over the age of 50 , it is still the most common cause of death in women between the ages of 40 and 50 years. There are a number of risk factors currently known ${ }^{1}$ and others under investigation. The most relevant risk factor in the present study is that of family history, long recognised to confer an increased risk. ${ }^{2}$ Up to $10 \%$ of breast cancer may be the result of a genetic predisposition. ${ }^{34}$ One of the genes (BRCA1) predisposing to breast (and ovarian) cancer was initially mapped to the long arm of chromosome 17 in $1990^{5}$ and cloned in $1994 .^{6}$ In a collaborative approach, the Breast Cancer Linkage Consor- tium (BCLC) ${ }^{7}$ showed that $B R C A 1$ mutations underlie approximately $50 \%$ of families with a clear dominant breast cancer predisposition and over $80 \%$ of those families in which both breast and ovarian cancer are present. A proportion of the remaining dominant families can be accounted for by the second breast cancer predisposing gene, $B R C A 2$ on chromosome 13q12-13, cloned in $1995 .{ }^{8}$

The BCLC used BRCA1 linked families to estimate that the incidence of breast cancer in $B R C A 1$ mutation carriers is $51 \%$ by the age of 50 and $85 \%$ by the age of 70 years. The risk of ovarian cancer was estimated at $63 \%$ by the age of 70 years. There is some evidence of increased risk of colon cancer in BRCA1 carriers of both sexes and prostate cancer in male carriers.

Genetic testing of affected women has been limited by the complexity of analysing a large gene with numerous unique mutations. Recently, however, the Ashkenazi Jewish population has been found to have a single mutation in BRCA1 occurring in up to $1 \%$ of their population, ${ }^{9}$ raising the possibility of easier access to widespread testing of both affected women and those at risk. However, since the majority of breast cancer cases are attributed to non-heritable factors, most women with a family history are not at sufficiently high risk to necessitate presymptomatic genetic testing.

This background of increasing genetic knowledge has contributed to pressure for the development and evaluation of appropriate service provision for women with a family history of breast cancer. ${ }^{10-12}$ However, the characteristics of the population likely to present to family history clinics are unclear, and despite the increased consumer "demand" no evidence of consumer "need" has yet been found. Some authors have called for large scale, prospective studies to evaluate the psychosocial and economic costs and benefits of receiving specialist genetic assessment for breast cancer susceptibility. ${ }^{13}$ Despite the lack of evidence, "services" are developing throughout the United Kingdom in different forms, in some centres research based and in other centres service clinics. It has been suggested that the ideal family history clinic would reflect an integrated approach involving surgeons, oncologists, and geneticists. ${ }^{14}$ With developments pending regarding the Calman-Hine Report ${ }^{10}$ and the recent Department of Health Report. ${ }^{12}$ it is timely to consider how cancer genetics services can be integrated. 


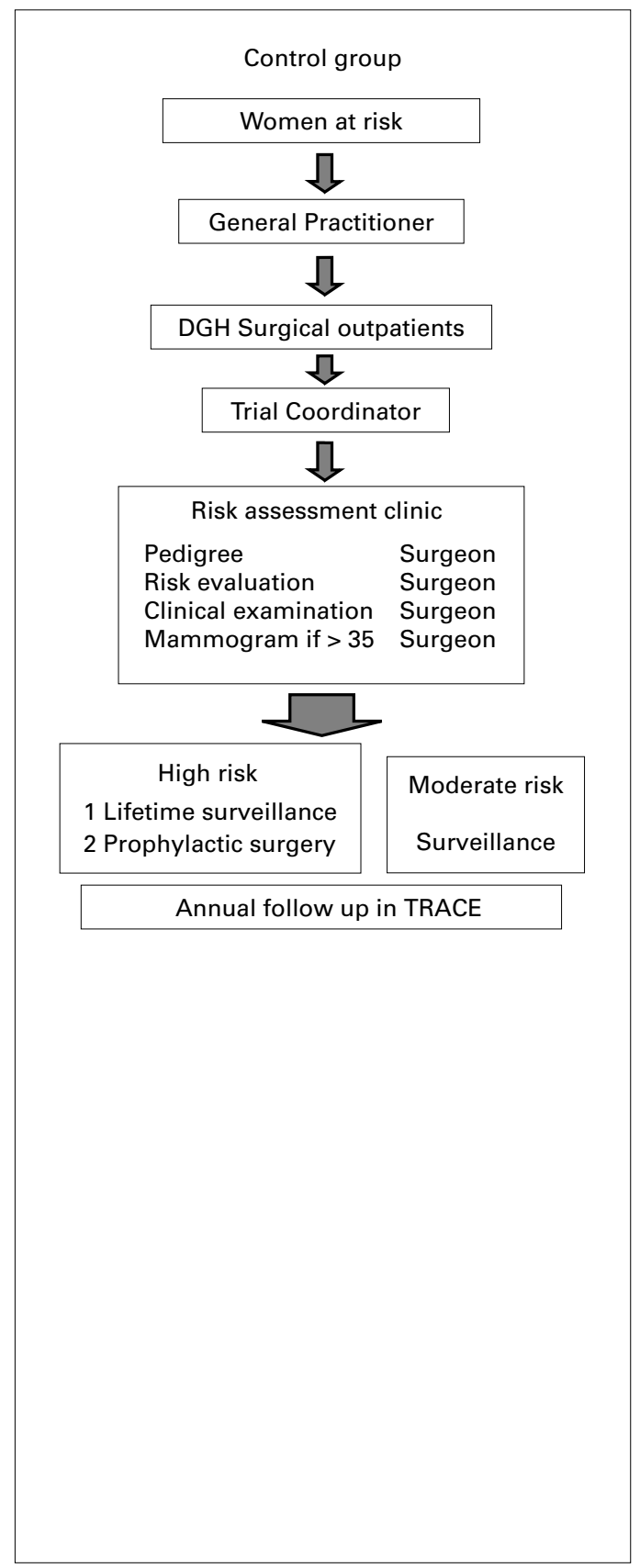

Figure 1 TRACE flow diagram.

The TRACE project (Trial of genetic assessment in breast cancer) is a randomised controlled trial of genetic assessment for breast cancer susceptibility. The objectives of the study include identifying the client population of women with a family history of breast cancer, and examining the psychosocial and resource implications of a multidisciplinary genetic and surgical assessment clinic for at risk women (fig 1).

Plan of investigation

A multidisciplinary assessment service for women with a family history of breast cancer (involving breast surgeons, nurse specialists, and genetics staff) was established to allow a randomised comparison with the existing clinical service, and was evaluated in terms of psy-
Study group

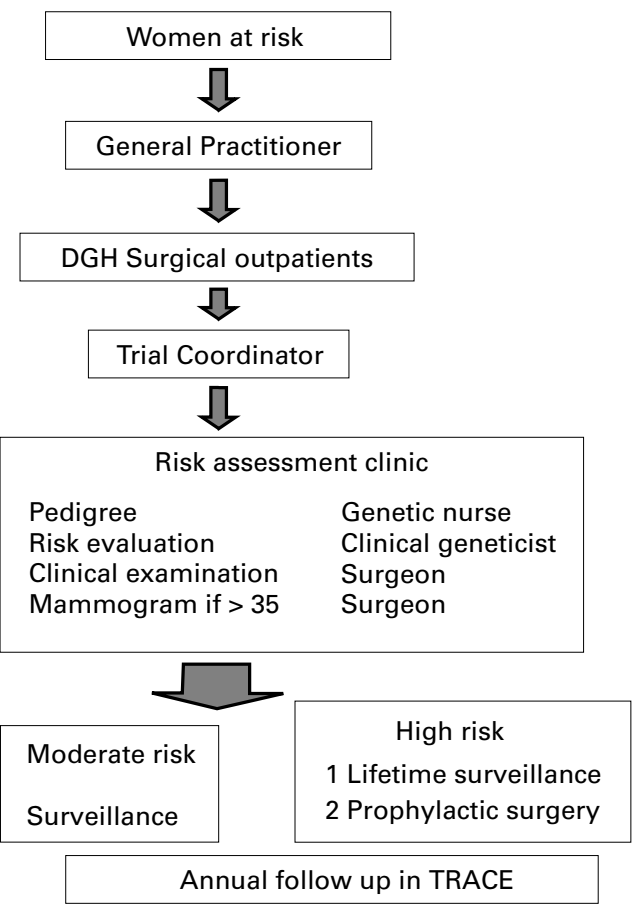

High risk suitable for presymptomatic testing if consent

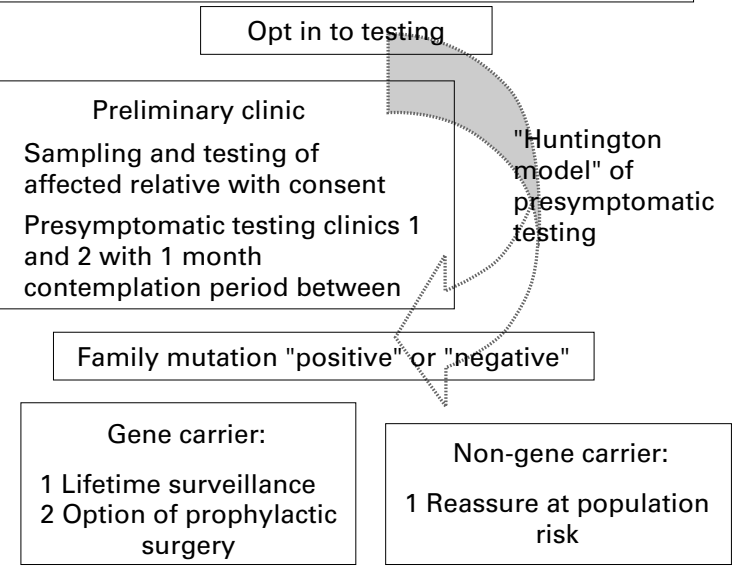

chosocial, health economic, and service delivery outcomes. Ethical approval for the project was obtained.

\section{Study entry criteria}

The study entry criteria were as follows. (1) Breast cancer in mother, sister, or daughter diagnosed under the age of 50 years. (2) Mother, sister, or daughter with bilateral breast cancer. (3) Breast cancer in two first degree relatives or a first and second degree relative. (4) Resident in Wales (total population 2.9 million). (5) No previous genetic counselling.

\section{Methods}

CONSENT

An information sheet and consent form were sent to women on receipt of a referral form 
from the District General Hospital (DGH). Women who felt unable to sign the consent form definitively before their clinic appointment were asked to attend and time was allocated to discuss the issues.

\section{STUDY DESIGN AND PROCEDURES}

All general practitioners (GPs) in Wales (total 1700) were circulated with information about the study before the start of recruitment. The information sent to GPs advised that women who were concerned about a possible family history of breast cancer and who fulfilled the study entry criteria could be referred to local specialist breast surgeons. Eligible women were given a brief written description of the study by the DGH surgeon, and those who verbally consented to participate were referred to the study. Before referral, the surgeon carried out a breast examination in order to exclude any palpable lumps. On receiving the referral form from the DGH surgeon, a package which included a baseline questionnaire, information sheet, and consent form was sent to women. The baseline questionnaire assessed demographic and psychological variables. Randomisation to the trial group or control group took place on receipt of the completed baseline questionnaire. Two clinic venues were chosen for the study, Breast Test Wales (BTW) Screening Centre and the existing family history clinic at the University Hospital of Wales (UHW), both in Cardiff. A four way randomisation was conducted according to a predetermined random allocation scheme, representing each combination of clinic type (trial and control) and clinic venue (BTW and UHW). This approach aided in controlling for intrinsic differences that existed between the two sites. In order to avoid possible response bias, there was no reference to the group allocation in either the baseline questionnaire or initial appointment letter. Participants were asked to complete and return further questionnaires to assess psychological outcomes. These were administered at two follow up points, immediately after clinic and nine months after clinic for women in both study groups. Follow up at nine months was chosen in order to avoid any overlap with annual surgical review, which was provided one year after clinic to women in both study groups.

\section{Control group}

Women who were randomly allocated to the control group were seen in a typical family history clinic, with clinical input provided by surgical staff including a breast surgeon and breast care nurse. The breast surgeon adhered to a standard protocol that included the following elements: (1) systematic clinical breast examination and, for women over 35 years of age, a mammogram; (2) surgical assessment of individual breast cancer risk presented in qualitative form and based on non-genetic information including age, reproductive history, and minimal family history; (3) the option of entering a chemoprevention trial; (4) advice about breast cancer surveillance and risk management; and (5) annual surgical review involving appropriate breast screening and advice. Referral for genetic counselling or presymptomatic genetic testing was not offered.

\section{Trial group}

Women randomly assigned to the trial group received a newly developed multidisciplinary genetic assessment service. This combined clinical input from surgical staff with a genetics consultation provided by genetics staff including a clinical geneticist and genetic nurse specialist. The central element of the trial condition was the provision of genetic assessment, involving individualised genetic risk assessment and the offer of presymptomatic testing for the $B R C A 1 / B R C A 2$ gene in women identified as "high" risk ( $\geqslant 25 \%$ residual lifetime risk of breast cancer) and who had a living affected relative. Surgical input was provided as previously, with individualised risk assessment provided instead by genetics staff who adhered to a standard protocol. Detailed family histories were obtained in which cancer related diagnoses were documented, with the aim of constructing and analysing a family pedigree for genetic risk assessment and counselling. Calculations of individualised breast cancer risk were made from pedigree data and analysed by the geneticist using the model developed by Claus et $a l^{15}$ incorporated into the Cyrillic pedigree drawing package. ${ }^{16}$ The variables included in this model are the number of first degree and second degree relatives affected with breast cancer and their age at diagnosis. Risk of breast cancer was presented in quantitative form (percentage residual lifetime risk) and compared to figures estimated for women of the same age in the general population. Genetic aspects of familial breast cancer were also discussed. A standard follow up letter that summarised details of the trial group consultation was sent to patients and referring surgeons.

Presymptomatic testing was offered using a model recommended by the UK Predictive Testing Consortium for Huntington's Disease (HD $)^{17}$ and developed at the Institute of Medical Genetics in Cardiff.$^{18}$ Women at high risk were offered a preliminary appointment at which medical, psychosocial, ethical, and legal issues were discussed. Consent forms were given to the consultand to pass to the affected relative, asking permission to view their medical notes and to make contact. If the consent form was returned, the genetic nurse specialist made contact with the affected relative, obtained fully informed consent for blood sampling, and initiated mutation searching. The advantage of identifying the mutation in a living affected relative is that the consultand's subsequent testing becomes a relatively clear presence or absence of the mutation known in that family. Once the mutation is identified in a family, at risk members who wish to be tested are offered two genetic counselling sessions separated by a one month contemplation period. The genetic counselling sessions cover a range of emotional as well as clinical issues. Women are also offered a standard clinical follow up protocol that is observed at the Institute 
of Medical Genetics, involving a telephone call one week after test results and a clinic appointment one month after results for everyone, and a yearly clinic appointment for gene carriers.

\section{PARTICIPANTS}

Over an 18 month recruitment period, a total of 1172 women were referred into the study. A statistical power calculation had indicated that minimum sample sizes of 95 per study group would be necessary to detect a medium effect size $(d=0.5)$ with a power of $80 \%$ at a significance level of $\alpha=0.01$, using analyses of between groups differences. Therefore power efficiency of the study design was shown to be above recommended levels. ${ }^{19}$ Data from the first 1000 referrals were considered sufficient for the purposes of the psychosocial evaluation and are reported in the companion paper by Brain et al. Of the 1000 referrals, 833 returned the baseline questionnaire and were randomised to the trial group $(n=366)$ or control group $(n=369)$.

\section{Evaluations}

PSYCHOSOCIAL EVALUATION

The associated paper by Brain et al reports cross sectional data regarding the baseline demographic, medical, and psychological characteristics of women recruited to the study. Subsequent publications will report prospective data relating to psychological outcomes including general anxiety, cancer worry, perceived risk of breast cancer, breast cancer knowledge, satisfaction, attitudes towards genetic testing, and intentions regarding risk management.

HEALTH ECONOMIC EVALUATION

An economic evaluation was undertaken within the study to estimate the additional cost of providing a specialist genetic assessment service compared with current practice of a breast surgeon consultation. This included the time required by health professionals to counsel, contact, and visit affected relatives and to verify family histories. Differential use of mammography and other investigations was monitored. The cost of searching for genetic mutations was estimated, and the knock on effects for primary care (GP visits concerning fears of breast cancer) and secondary care (prophylactic mastectomy and increased lifetime surveillance) was monitored. Since the evaluation adopted a societal perspective, patient borne costs including travel, time off work, and child care costs were also recorded.

Conventional methods of valuation were used throughout, ${ }^{20}$ with sensitivity analyses undertaken to measure the robustness of results to changes in key variables. Assessing these costs against the full range of benefits will provide an indication of the efficiency of specialist genetic assessment. Since there are many demands being placed on NHS resources, it is important that information on the efficiency of the service be gleaned in addition to information on the effectiveness.

\section{Discussion}

The current randomised controlled trial allows evaluation of the potential benefits and disadvantages of adding genetic assessment to existing surgical management for women with a family history of breast cancer. However, we recognise the potential problems implicit in the study design. Firstly, there is the possibility of contamination of the surgical input into the control arm. There was always a possibility that the surgeons would increase in genetic knowledge through their involvement in the study and fail to continue to represent "typical" surgical breast specialists. Despite this potential problem, we felt it important to use the same surgeons in both the trial and control arms in order to minimise other variables.

Secondly, the offer of individualised risk assessment and presymptomatic testing are unique to the trial group and this raises questions of whether women should be allowed to switch between study groups. At the time the project was initiated, specific cancer genetics provision and BRCA1/BRCA2 testing was not available for families at increased risk of breast cancer in Wales. At that time, standard practice involved management at a surgical clinic only. The introduction of genetic assessment and possible testing was deemed to be unproven; accordingly, ethical permission for the randomised controlled trial was granted in Wales. Therefore, there was no facility for transferring across groups. However, women could opt to leave the study and therefore "leakage" to other centres was monitored. Numbers attending other centres for genetic testing during this period were very small, with final figures being collated by neighbouring genetic centres.

Thirdly, work conducted in recent years with new approaches to risk assessment and evaluation of $B R C A 1 / B R C A 2$ risk may raise questions regarding the present study referral criteria. In the present study, we opted to present risk as residual lifetime risk and to identify "high risk" subjects as those with $25 \%$, or greater, residual lifetime risk of breast cancer. We found residual lifetime risk a useful way of discussing risk with women in the trial arm of the study. In addition, the cut off point of $25 \%$ or greater residual lifetime risk gave a detection rate of over $20 \%$ of $B R C A 1 / B R C A 2$ mutations, as will be shown in subsequent publications.

The TRACE project was designed to provide information that will be of direct relevance to future service provision in relation to genetic aspects of breast cancer. The linked article by Brain et al identifies important characteristics of the presenting population. The next step is to assess the psychosocial and economic implications of adding a "specialist genetic" component to existing care for these women. Service delivery questions such as this need to be answered in order to allow the appropriate introduction of new genetic advances and to avoid ad hoc developments. The guidelines on appropriate cancer genetic services released by the Department of Health ${ }^{12}$ highlight possible service structures that should be purchased, and the TRACE project will provide information regarding patients' 
responses to receiving different elements of this structured care.

The TRACE project has relevance in other cancers. Bowel cancer is perhaps closer to a "service ready" situation, with better tumour detection and evidence of survival benefit from the screening of those at high risk. Apart from breast and bowel cancer, genetic advances will affect how we manage other common cancers, for example, prostate cancer. By debating the issues in relation to TRACE, it may be possible to identify sensible conclusions applicable to the whole group of disorders before media publicity and widespread public concern create unfocused "demand". The wider value of this debate goes beyond the field of cancer, since the same issues of a high risk genetic subset are now being found in an increasing range of disorders. Knowing what factors may make specialist genetic assessment important, and which situations do not need a specialist input, should help to target resources selectively where they are needed, while ensuring that the concerns of the vast majority without a strong genetic predisposition can be relieved.

We thank the Medical Research Council, Welsh Office, and NHS R\&D (Wales) for support of the TRACE project, Dr Cyril Chapman for his help with the Cyrillic computer package, and Imperial Cancer Research Fund for support of Liz France.

1 McPherson K, Steel CM, Dixon JM. Breast cancer - epidemiology, risk factors and genetics. BMf 1994;309:1003-6. 2 Anderson DE. A genetic study of human breast cancer. $\mathcal{F}$ Natl Cancer Inst 1972;48:1029-34.

3 Eeles R. Testing for the breast cancer predisposition gene BRCA1. BMF 1996;313:572.
4 King MC, Rowell S, Love SM. Inherited breast and ovarian cancers, what are the risks? What are the choices? $\mathcal{F A M A}$ cancers, what are the

5 Hall JM, Lee MK, Newman B, et al. Linkage of early onset familial breast cancer to chromosome 17q21. Science 1990; 250:1684-9

6 Miki Y, Swensen J, Shattuck-Eidens D, et al. A strong candidate for the breast and ovarian cancer susceptibility gene, BRCA1. Science 1994;266:66-71

7 Easton DF, Bishop DT, Ford D, Crockford GP, and the Breast Cancer Linkage Consortium. Genetic linkage analysis in familial breast and ovarian cancer: results from 214 families. Am f Hum Genet 1993;52:678-701.

8 Wooster R, Neuhausen S, Mangion J, et al. Localisation of a breast cancer susceptibility gene BRCA2 to chromosome 13q12-13. Science 1994;265:2088-90.

9 Struewing JP, Abehovich D, Peretz T, et al. The carrier frequency of the BRCA1 185delAG mutation is approximately 1 percent in Ashkenazi Jewish individuals. Nat Genet 1995;11:198-200.

10 Calman Hine Report. A policy framework for commissioning cancer services. Report by the expert advisory group on cancer to the Chief Medical Officers of England and Wales. London: Department of Health and Welsh Office, 1995.

11 Campbell H, Mackay J, Porteous M. The future of breast and ovarian cancer clinics. BMF. 1995;311:1584-5.

12 Genetics and Cancer Services. Report of a Working Group for the Department of Health, 1998.

13 Lerman C, Croyle R. Psychological issues in genetic testing for breast cancer susceptibility. Arch Intern Med 1994;154: 609-16

14 Lynch HT, Watson P, Conway TA, et al. DNA screening for breast/ovarian cancer susceptibility based on linked markers. Arch Intern Med 1993;153:1979-87.

15 Claus EB, Risch N, Thompson WD. Genetic analysis of breast cancer in the cancer and steroid hormone study. $\mathrm{Am}$ f Hum Genet 1991;48:232-42.

16 Chapman CJ. Cyrillic for pedigree drawing. Oxford: Cherwell Scientific Publishing, 1997.

17 IHA/WFN. Guidelines for the molecular genetic predictive test in Huntington's disease. F Med Genet 1994;31:555-9.

18 Ball D, Harper PS. Presymptomatic testing for late onset Ball D, Harper PS. Presymptomatic testing for late onset genetic disorders: lessons

19 Cohen J. Statistical power analysis for the behavioral sciences. 2nd ed. Hillsdale, New Jersey: Lawrence Erlbaum Associates, 1988.

20 Drummond M, Stoddart G, Torrance G. Methods for the economic evaluation of health care programs. Oxford: Oxford Medical Publications, 1997. 